ARTICLE

\title{
Ultrasensitive antibody-aptamer plasmonic biosensor for malaria biomarker detection in whole blood
}

\author{
Antonio Minopoli (10) 1,2, Bartolomeo Della Ventura², Bohdan Lenyk (1) 1,3, Francesco Gentile ${ }^{4}$, Julian A. Tanner (1) ${ }^{5}$, \\ Andreas Offenhäusser ${ }^{1}$, Dirk Mayer (i) ${ }^{1 凶} \&$ Raffaele Velotta (i) ${ }^{2 凶}$
}

Development of plasmonic biosensors combining reliability and ease of use is still a challenge. Gold nanoparticle arrays made by block copolymer micelle nanolithography (BCMN) stand out for their scalability, cost-effectiveness and tunable plasmonic properties, making them ideal substrates for fluorescence enhancement. Here, we describe a plasmon-enhanced fluorescence immunosensor for the specific and ultrasensitive detection of Plasmodium falciparum lactate dehydrogenase ( $P f L D H)-a$ malaria marker-in whole blood. Analyte recognition is realized by oriented antibodies immobilized in a close-packed configuration via the photochemical immobilization technique (PIT), with a top bioreceptor of nucleic acid aptamers recognizing a different surface of $P f L D H$ in a sandwich conformation. The combination of BCMN and PIT enabled maximum control over the nanoparticle size and lattice constant as well as the distance of the fluorophore from the sensing surface. The device achieved a limit of detection smaller than $1 \mathrm{pg} / \mathrm{mL}(<30 \mathrm{fM})$ with very high specificity without any sample pretreatment. This limit of detection is several orders of magnitude lower than that found in malaria rapid diagnostic tests or even commercial ELISA kits. Thanks to its overall dimensions, ease of use and high-throughput analysis, the device can be used as a substrate in automated multi-well plate readers and improve the efficiency of conventional fluorescence immunoassays.

\footnotetext{
${ }^{1}$ Institute of Biological Information Processing (IBI-3), Bioelectronics, Forschungszentrum Jülich, 52425 Jülich, Germany. ${ }^{2}$ Department of Physics "E. Pancini", University of Naples "Federico II", Via Cintia 26, 80126 Naples, Italy. ${ }^{3}$ Department of Physics, University of Konstanz, 78457 Konstanz, Germany.

${ }^{4}$ Department of Experimental and Clinical Medicine, University Magna Graecia, 88100 Catanzaro, Italy. ${ }^{5}$ School of Biomedical Sciences, University of

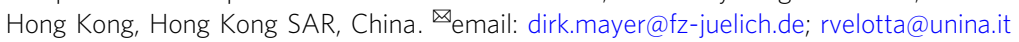


F luorescence-based techniques are among the most widespread in the fields of biotechnology, life sciences, and biomedical research ${ }^{1-3}$. Fluorescent probes and fluorescentlabeled bioreceptors are extensively employed in optogenetic studies $^{4}$, cytogenetic assays, in fluorescent in situ hybridization ${ }^{5}$, bioimaging analysis, for the observation of both the location and motion of cells or subcellular elements ${ }^{6}$, molecular dynamic investigation ${ }^{7}$, as well as in fluoroimmunoassays for the detection of molecular biomarkers and in enzyme-linked immunosorbent assay (ELISA) ${ }^{8}$. However, the poor yield of many fluorescent dyes and signal interference can hamper the application of fluorescence to the field of sensing. The detection of analytes in very low abundance ranges is a long-standing goal of fluorescence. Many efforts have been carried out to enhance the fluorescent signal in order to extend their application to ultra-sensitive fluoroimmunoassays ${ }^{9,10}$.

Plasmonic nanostructures are realistic candidates to extend the limit of fluorescence detection to the femtomolar level and beyond $^{11,12}$. These devices modify the spectral properties of the nearby fluorescent dyes and do not necessitate expensive equipment, specific or toxic reagents, or significant modifications to well-established fluorescence-based assays. Such a modification depends strongly both on the spectral overlap between the fluorescent dye and the plasmon absorbance ${ }^{13,14}$ and on the fluorophore-nanostructure distance $z^{15-17}$. In particular, if the plasmon absorbance overlaps the fluorophore excitation peak, a strong fluorescence enhancement (FE) is achieved for small values of $z(<10 \mathrm{~nm})$ (up to 100 -fold $\left.{ }^{17,18}\right)$, thanks to the Förster resonance energy transfer (FRET) mechanism, whereas a weak coupling occurring at higher values of $z$ leads to a vanishingly small $\mathrm{FE}^{14,16}$. In contrast, the overlap with the fluorophore emission peak provides a large FE for high $z(>10 \mathrm{~nm})$, thanks to the enhancement of the fluorophore radiative rate through the Purcell effect, while a progressive decrease of the FE occurs at smaller $z$ 's, due to the increasing fluorescence quenching via nonradiative losses in the metal nanostructure ${ }^{14,16}$. In the case that the plasmon absorbance superimposes over both the excitation and emission peak of the fluorophore, i.e., dual-mechanism enhancement $^{19}$, a huge $\mathrm{FE}$ arises at an optimal distance of $\sim 10-15 \mathrm{~nm}$, a strong quenching being present at shorter $z$ 's, whereas a return to the no enhanced fluorescence conditions occurs at longer distance due to the weaker plasmon-fluorophore coupling ${ }^{14,16-18}$.

Two-dimensional (2D) arrays of metal nanostructures are particularly suitable as plasmon-enhanced fluorescence (PEF)based biosensing platforms ${ }^{12}$. Until now, a variety of fluorescence enhancers, such as gold micro-islands ${ }^{20}$, arrays of metal nanoobjects (e.g., nanoparticles ${ }^{21}$, nanorods ${ }^{22}$, nanotriangles ${ }^{23}$, nanocrystals $\left.^{24}\right)$, bow-tie nanoantennas ${ }^{25}$ and resonant nanocavities ${ }^{26,27}$, have been exploited to push down the detection limit in fluorescence-based assays. Despite significant milestones in terms of limit of detection (LOD) (down to $\mathrm{fM}$ level ${ }^{28,29}$ ), FE factor (up to $10^{5}$-fold ${ }^{27,30}$ ) and adaptability to the conventional and well-established fluoroassays ${ }^{31,32}$, still a number of factors limit the use of those solutions as devices for routine testing, point-of-care analysis and large-scale use of the PEF-based fluoroassays, including, to cite a few, the need for skilled personnel, expensive technologies, time-consuming procedures, poor versatility in tuning of plasmon-fluorophore coupling and not easy scalability ${ }^{9}$.

2D-lattice of gold nanoparticles (AuNPs), made by block copolymer micelle nanolithography $(\mathrm{BCMN})^{33}$, provide an easy way to overcome most of the aforementioned challenges. The affordable and scalable fabrication and the facile tunability of their plasmonic properties are the main strengths of these devices $^{33}$. The plasmonic behavior of a 2D AuNP lattice is closely related to the ratio $R$ between the nanoparticle diameter $D$ and the interparticle distance $d$. When a nanoparticle is placed in close proximity to its nearest neighbors $(R>2 / 3)^{34}$, interaction among the localized surface plasmons (LSPs) gives rise to longrange collective oscillation ${ }^{35,36}$. Such a collective effect is negligible if $R<2 / 3$, in which case the plasmonic response of the 2Dlattice is well-described by a system of decoupled $\operatorname{LSPs}^{34}$.

A key issue to address when such plasmonic substrates are used in biosensing is the biofunctionalization of the active surface since it deeply affects both the sensitivity and specificity ${ }^{37}$. Antibodies (Abs) are the prime candidates as bioreceptors thanks to their inherent specificity, versatility, and reliability. However, due to their moderate long-time stability and the need to immobilize them both with the right orientation and high surface density, the accomplishment of a robust and effective $\mathrm{Ab}$ surface functionalization is still an open issue ${ }^{38}$. In this respect, the well-established photochemical immobilization technique (PIT) is a simple, fast and effective strategy to tether Abs onto gold surfaces with one fragment antigen-binding (Fab) exposed to the surrounding environment ${ }^{39}$. Besides antibodies, aptamers are nucleic acids which have been evolved to bind to targets through molecular evolution approaches as alternative class of bioreceptors. Combining aptamers and antibodies has been proven as a powerful approach for molecular recognition ${ }^{40}$, but such approaches have not been investigated for PEF-based sensors.

In the present work, we describe an ultrasensitive and costeffective PEF-based immunosensor-consisting of 2D AuNP array functionalized by PIT-able to detect proteins at femtomolar level in whole human blood. Specifically, we measured the concentration of Plasmodium falciparum lactate dehydrogenase $(P f L D H)$, a malaria biomarker present at nanomolar level in red blood cells but only at picomolar levels in serum of infected people $\mathrm{e}^{41}$. Malaria is still one of the main causes of disease-related deaths worldwide ${ }^{42,43}$ and it is caused by Plasmodium parasites such as $P$. vivax and $P$. falciparum, the latter accounting for $90 \%$ of mortality worldwide ${ }^{44}$. The conventional serologic antibodybased rapid diagnostic tests rely upon the PfLDH detection in pretreated blood and offer rapid and cost-effective malaria diagnosis. However, the poor LOD as well as the transportation and storage difficulties of pre-functionalized devices in tropical environment make such tests unfit for early diagnosis and screening ${ }^{45-47}$. The ultrasensitive PEF-based device in combination with a unique functionalization procedure (PIT), which can be accomplished in a few minutes, results in an immunosensor suitable for detecting PfLDH at femtomolar level in the whole blood without any pretreatment and preconcentration steps. The fluoroimmunoassay proposed here is inherently specific since the recognition of $P f L D H$ takes place in a sandwich scheme by Abs from the bottom and aptamers from the top; thus, it can be immediately extended to other analytes by replacing the bioreceptor layers.

\section{Results}

Fluorescent aptamer-based immunoassay. BCMN was employed to produce arrays of ordered AuNPs with tunable density, size, and interparticle distance ${ }^{33}$. Diblock copolymers with amphiphilic character were dispersed in a non-polar solvent (toluene) obtaining reverse micelles with a spherical shape, a hydrophilic core, and an outer hydrophobic shell (Fig. 1a). The resulting micelle can house the gold precursor inside, allowing the formation of AuNPs covered by a hydrophobic shell (PS-AuNPs). The substrates were then dipped into a solution containing PSAuNPs by a dip coater to ensure a careful tuning of the dipping speed. The PS-AuNPs were transferred on the non-polar glass surface by hydrophobic interaction giving rise to a self-assembled 
a

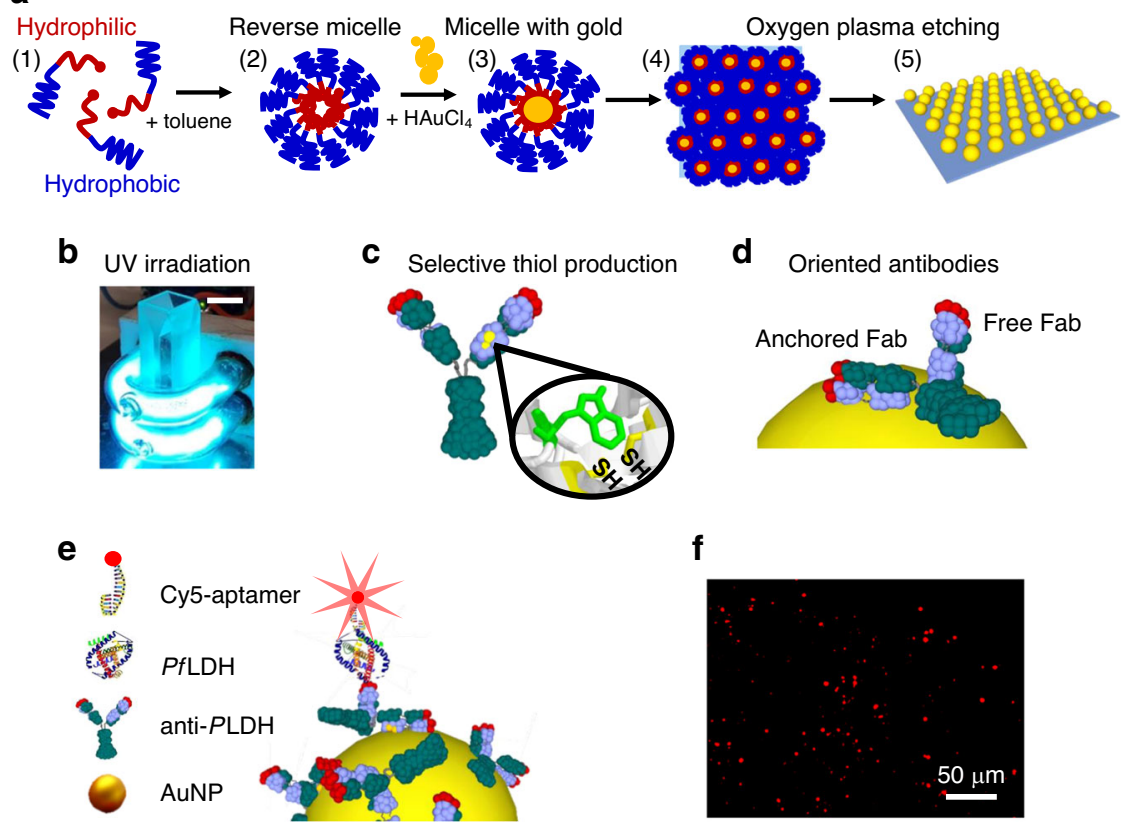

Fig. 1 Operating principle of the device. a Fabrication process of AuNP array by BCMN: (1) dispersion of diblock copolymers with amphiphilic character in toluene solution; (2) formation of reverse micelles with hydrophilic core and outer hydrophobic shell; (3) loading of the gold precursor inside the micelles; (4) sticking of the PS-AuNPs on the substrate through hydrophobic interaction; (5) immobilization of the AuNPs on the substrate after copolymer etching. b Low pressure mercury U-shaped UV lamps used to carry out the biofunctionalization of AuNPs with antibodies through PIT. A standard 10 mm cuvette can be easily housed inside the internal volume (the length of the scale bar in the top-right corner is $1 \mathrm{~cm}$ ). Given the proximity of the cuvette to the lamps and the wrapping geometry, we estimated that the solution was exposed to an UV-irradiation of $0.3 \mathrm{~W} / \mathrm{cm}^{2}$. c UV irradiation of the Abs leads to the production of four thiol groups (two of them are not visible in the figure). $\mathbf{d}$ The position of the thiols, opposite with respect to the plane containing the antibody Fabs, allows to immobilize the Abs with one of their binding sites exposed to the surrounding environment. e Sketch of the Ab-PfLDH-aptamer sandwich scheme used throughout the experiments. The adoption of Abs as bottom receptor layer and aptamers as top layer allows the fluorophore to be placed at a distance of approximately $10 \mathrm{~nm}$ from the surface. $\mathbf{f}$ Example of fluorescence picture acquired at $1 \mathrm{pM}(35 \mathrm{pg} / \mathrm{mL}) P f \mathrm{LDH}$ concentration. The red spots arise from the fluorescence emitted by a single Cy5 molecule.

close-packing honeycomb arrangement. Then, the copolymers were etched by oxygen plasma treatment leaving the AuNPs immobilized on the glass surface at predetermined positions (Fig. 1a). Subsequent incubation in a gold solution entailed the growth of the AuNP size ${ }^{48}$. The resulting reduction of interparticle distance brought about an increase of the ratio $R$ up to a value of 2.5 that is large enough to activate collective plasmonic effects of the $\operatorname{array}^{34,36}$.

Gold NPs on the surface were functionalized with pan malaria antibodies (anti-PLDH) using PIT ${ }^{39,49,50}$. Such a technique consists of UV irradiation of Abs (Fig. 1b) that leads the selective photoreduction of the disulfide bridge in specific cysteine-cysteine/tryptophan (Cys-Cys/Trp) triads ${ }^{51}$. The breakage of such Cys-Cys bonds in both Ab Fab fragments produces four free thiol groups (Fig. 1c), two of which are able to interact with the proximal gold surface giving rise to a covalent Ab tether. Functionalization by PIT assures control over the orientation of the immobilized Abs, with one of their binding sites exposed to the surrounding environment ${ }^{39}$ (Fig. 1d). Moreover, the technique leads to a close-packing arrangement of the $\mathrm{Abs}^{52,53}$, demonstrated by the plasmon resonance of the device. In experiments where we varied the concentration of the anti$P L D H$ over large intervals (see Supplementary Fig. 1), the wavelength shift $\left(\Delta \lambda_{\mathrm{p}}\right)$ showed no significant variations for concentrations larger than $50 \mu \mathrm{g} / \mathrm{mL}$, that is a threshold above which no more free AuNP surface is available for Abs functionalization. We therefore used the value of concentration $50 \mu \mathrm{g} / \mathrm{mL}$ throughout the work. The close-packed biofunctionalization is also evident by the lack of $\Delta \lambda_{p}$ changes after the blocking step (Supplementary Fig. 2). We estimated the value of $\mathrm{Ab}$ density (number of Abs per AuNP) as $\sim 50$, which corresponds to the maximum number of antibodies that can be anchored onto a sphere of diameter $50 \mathrm{~nm}$ (the steric hindrance of a single immobilized $\mathrm{Ab}$ is $\left.\sim 150 \mathrm{~nm}^{2}\right)^{52}$.

Figure 1e shows the sandwich scheme used for the immunosensor. The bottom bioreceptor layer (anti-PLDH) provides a reliable and effective targeting of any malaria biomarkers Plasmodium lactate dehydrogenase $(P L D H)^{54}$, whereas the top bioreceptor layer (aptamers) provides a cheap and versatile labeling of the analyte with fluorescent tag and ensures an extremely high specificity against PfLDH ${ }^{55}$. Aptamers in the top layer enable the fluorophore to be placed at an optimal distance from the AuNP of $\sim 10 \mathrm{~nm}$, assuring optimal PEF amplification ${ }^{14,15}$. The fluorescence signal was recorded by a fluorescence microscope and the resulting pictures were analyzed by an image processing software to retrieve the corresponding intensity. Figure if shows a typically processed image collected at low PfLDH concentration, in which the original non-flat background was removed by using the "rolling ball" algorithm ${ }^{56}$ (see Supplementary Fig. 3) and the resulting red spots arose from photons emitted by a single fluorophore.

Plasmonic response of a honeycomb lattice. We used a numerical finite difference scheme implemented in Lumerical to simulate the electromagnetic (EM)-field scattered by the gold spheres on the sensor surface. The "FDTD solutions" tool of Lumerical software works out the numerical solutions of the Maxwell's equations via the finite-difference time-domain 
a

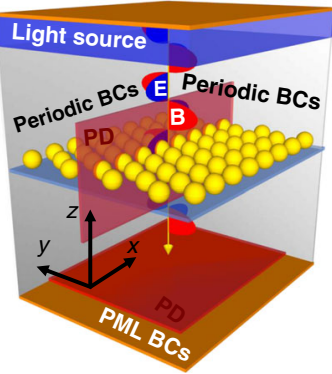

C

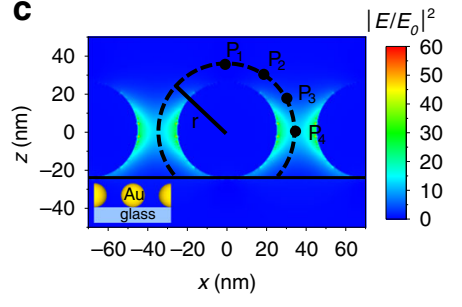

b

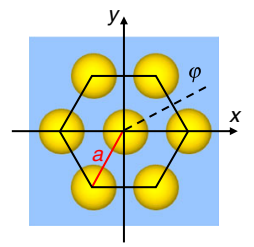

d

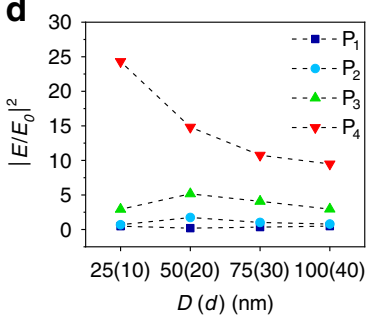

Fig. 2 Optical properties of 2D AuNP lattice. a Schematic representation of the simulation workspace consisting of plane wave source, plasmonic nanostructure, dielectric substrate $\left(\mathrm{SiO}_{2}\right.$ glass), photodetectors and appropriate $\mathrm{BCs}$. Linearly $x$-polarized $\mathrm{EM}$ wave propagates through the $2 \mathrm{D}$ AuNP lattice. The transmitted photons are collected by a PD on the opposite side of the workspace, whereas the E-field intensity distribution is worked out by placing several PDs in correspondence of the lattice (not all depicted in the sketch). b Top view sketch of the simulation unit cell; the centers of the spherical AuNPs belong to the plane $z=0$. The parameters $a$ and $\varphi$ are the lattice constant and the azimuthal angle, respectively. c Example of E-field distribution normalized to the incident radiation worked out in the plane $y=0$ for $x$-polarized EM plane wave incident on a lattice made of AuNPs with diameter $50 \mathrm{~nm}$ and interparticle distance $20 \mathrm{~nm}$. The points $\mathrm{P}_{1}-\mathrm{P}_{4}$ are at a distance of $10 \mathrm{~nm}$ from the nanoparticle surface at polar angle $\theta=0^{\circ}, 30^{\circ}, 60^{\circ}$, and $90^{\circ}$, respectively. $\mathbf{d}$ Gain of the E-field intensity as a function of AuNP diameter (interparticle distance).

(FDTD) method within a Mie problem-like workspace. A schematic representation of the simulation workspace and unit cell are shown in Fig. 2a, b, respectively. The electric field E (E-field) intensity exhibits a maximum in the $x-y$ plane along the polarization direction, while it shows a minimum in the transverse direction (see Supplementary Fig. 4).

By rotating the direction of polarization around $z$, we observed an azimuthal E-field intensity modulation with an expected period of $60^{\circ}$. Since all the polarization directions have to be taken into account when non-polarized light is used (e.g., light emitted by a fluorophore), the actual local E-field intensity is the result of the average of all such contributions (see Supplementary Fig. 5a). It turns out that the gain factor $\left|E / E_{0}\right|^{2}$ slightly modulates around a value of 4 when $\varphi$ is changed from $0^{\circ}$ to $360^{\circ}$ and the distance is $10 \mathrm{~nm}$ from the nanoparticle surface (see Supplementary Fig. 5b), thereby suggesting that the E-field intensity experienced by the fluorophore has a relatively weak azimuthal dependence.

The analysis as a function of the polar angle $\theta$ is reported in Fig. $2 c$ that shows the distribution of the E-field intensity in the $y=0$ plane, resulting from the interaction of an $x$-polarized plane wave with a $2 \mathrm{D}$ honeycomb lattice (Fig. 2b) made of $50 \mathrm{~nm}$ AuNPs distanced $20 \mathrm{~nm}$ one from the other. Due to the plasmon coupling, the largest values of the field intensity are recorded along the direction of polarization $(x)$ while the smallest values are measured in the transverse direction to the plane $z=0$. In

particular, the gain is more effective in the interval $45^{\circ} \leq \theta \leq 135^{\circ}$ that contains approximately $70 \%$ of the available surface.

Aiming at optimizing the performance of the substrate, we conducted a test-campaign where the response of the system was analyzed as a function of the characteristics of the 2D lattice. In the simulations, we varied the particle size and interparticle distance over large intervals $(25 \mathrm{~nm} / 10 \mathrm{~nm}, 50 \mathrm{~nm} / 20 \mathrm{~nm}, 75 \mathrm{~nm} /$ $30 \mathrm{~nm}, 100 \mathrm{~nm} / 40 \mathrm{~nm}$ ) keeping constant their ratio $R=2.5$, a value that is large enough to warrant a high enhancement factor $^{34,36}$. Figure $2 \mathrm{~d}$ shows the enhancement of the E-field intensity for several polar angles $\left(\theta=0^{\circ}, 30^{\circ}, 60^{\circ}, 90^{\circ}\right)$ at a distance of $10 \mathrm{~nm}$ from the AuNP surface (points $\mathrm{P}_{1}-\mathrm{P}_{4}$ in Fig. 2c). Despite the strongest gain was achieved for the smallest nanoparticles, the need to allow the formation of the Ab-PfLDHaptamer sandwiches in the interparticle gaps suggested us to consider $50 \mathrm{~nm} / 20 \mathrm{~nm}$ as the optimal choice to guarantee good enhancement of the E-field intensity while assuring that the process of functionalization and detection occurs correctly.

Optical and morphological characterization of the 2D AuNP array. The characterization of the substrate was performed by scanning electron microscopy (SEM) and UV-Vis spectroscopy (Fig. 3). SEM images of the device (Fig. 3a, b) show the fabrication process capability to attain maximum control over the geometrical characteristics of the device, including particle size and particle distance. Aiming at realizing a substrate with a unique collective plasmonic behavior, the nanoparticle growth is carried out to increase the $R$ value (Fig. 3c). The nanoparticle diameter increased approximately five-fold while the interparticle distance reduced three-fold by holding the lattice period equal to $\sim 70 \mathrm{~nm}$. The $R$ value went from 0.17 to 2.5 warranting a collective response of the AuNPs immobilized on the substrate 34,36 . The size distribution of the AuNPs before the gold growth process (blue columns) is peaked at approximately $10.4 \mathrm{~nm}$ with a full width at half maximum (FWHM) of $1.4 \mathrm{~nm}$, while that after nanoparticle growth (red columns) has a mean of $\sim 48 \mathrm{~nm}$ and a FWHM of $6 \mathrm{~nm}$ (Fig. 3d). The smaller peak at approximately $61 \mathrm{~nm}$ (red columns) is due to fewer AuNP clusters as a byproduct of the gold nanoparticle growth process. The center-to-center distance $d_{\mathrm{C}-\mathrm{C}}$, measured as the distance of each AuNP centroid with its nearest neighbors follows the distributions in Fig. $3 \mathrm{e}$. The mean values $\bar{d}_{\mathrm{C}-\mathrm{C}}$ are $69 \mathrm{~nm}$ (blue columns) and $68 \mathrm{~nm}$ (red columns) with standard deviations of $8 \mathrm{~nm}$ and $14 \mathrm{~nm}$, respectively. The high similarity of such distributions confirms the holding of most of AuNP positions also after the growth process, whereas the relatively large values of standard deviation for $\bar{d}_{\mathrm{C}-\mathrm{C}}$ can be ascribed to defects, such as clusters and vacancies. The occurrence of $d_{\mathrm{C}-\mathrm{C}}$ lower than $D$ after the growing process (red histograms in Fig. 3d, e) is due to the lack of the AuNP spherical shape arising from nanoparticle clustering (Fig. 3b).

The 2D AuNP array was also characterized optically by measuring its extinction spectrum whose resonance wavelength and shape are not only strongly dependent on the ratio $R^{34,57}$, but also on the regularity holding at high macroscopic level, a feature warranted by the array fabrication procedure adopted in this work (see Supplementary Fig. 6 for SEM pictures on large scale). Figure $3 \mathrm{f}$ shows a plasmonic resonance (green continuous line) occurring at approximately $650 \mathrm{~nm}$ whose width is large enough to contain both the excitation (blue dotted curve) and emission (red dashed curve) spectra of cyanine 5 (Cy5), fluorophore used in this experiment, which has an excitation peak at $649 \mathrm{~nm}$, an emission peak at $666 \mathrm{~nm}$ and a quantum yield (QY) of $0.27^{58}$. The superposition of the plasmonic resonance to both excitation and emission peaks of the fluorophore provides an ideal condition for achieving a PEF amplification since both FRET and Purcell effect 

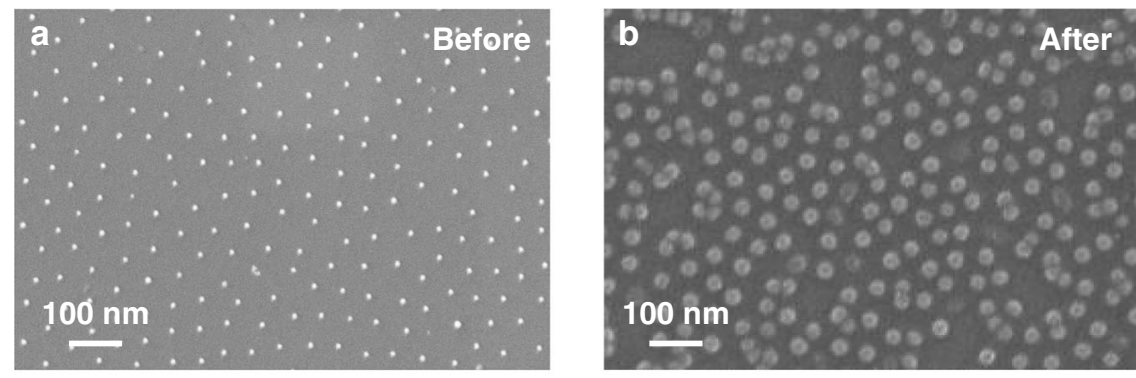

C
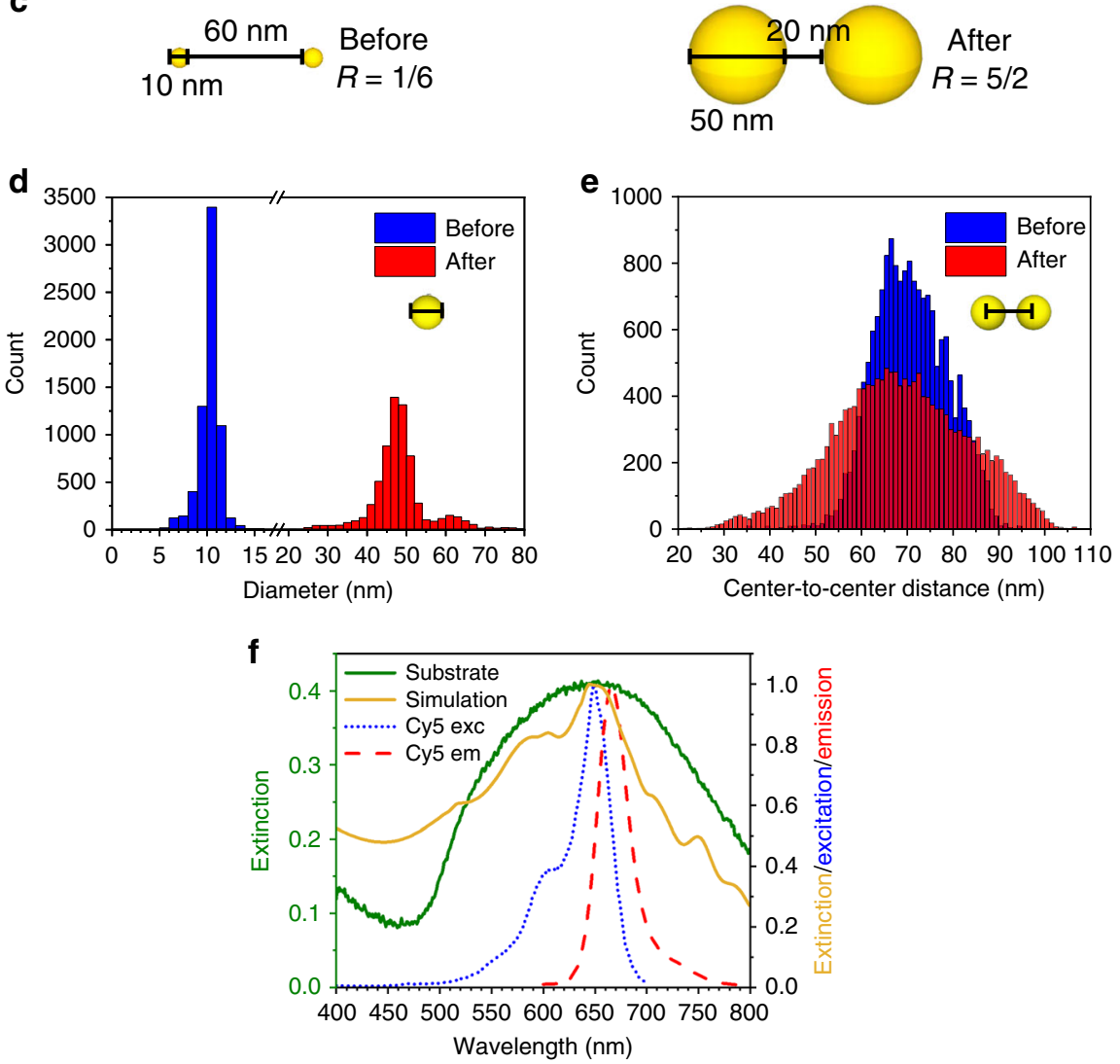

Fig. 3 Substrate characterization. a, b Top view SEM images of the AuNP array show high regularity of nanoparticle shape and size. Defects arising during the AuNP growth step, such as clusters and holes are randomly distributed on the substrate. c Sketch of the AuNP growth process. The nanoparticle diameter increases approximately five-fold while the interparticle distance reduces three-fold by holding the lattice period (center-to-center distance among nearest neighbors) equal to $\sim 70 \mathrm{~nm}$. The $R$ value goes from $\sim 0.17$ to $\sim 2.5$ warranting a collective plasmonic behavior of the AuNPs immobilized on the substrate. $\mathbf{d}$ Histograms of nanoparticle diameter before (blue columns) and after (red columns) incubation with gold growth solution. The distributions are peaked at $\sim 11 \mathrm{~nm}$ and $\sim 48 \mathrm{~nm}$, respectively. e Center-to-center distance histograms exhibit similar distributions confirming the holding of most of AuNP positions also after the gold growth process. The wider distribution delivered by the substrates after the AuNP growth (red columns) is due to the presence of lattice irregularities and defects arising during the incubation process. $\mathbf{f}$ The experimental extinction spectrum of the substrate (green continuous line) is well reproduced by simulating the optical response provided by the actual substrate morphology as measured by SEM (gold continuous line). The excitation and emission peaks of $\mathrm{Cy} 5$ (dotted blue line and dashed red line, respectively) are encompassed within the substrate plasmon resonance.

can take place ${ }^{11,13,14}$. Such a superposition can be easily realized at essentially any substrate plasmon resonance given the wide variety of fluorescent dyes apt to be used as aptamer label. The experimental extinction spectrum is well reproduced by that one worked out by considering the real morphology of the substrate as provided by SEM (gold continuous line), the latter being obtained by averaging the extinction spectra resulting from fifteen regions of interest sampled from the SEM image shown in Supplementary Fig. 7a (an example of the rendering provided by Lumerical is shown in Supplementary Fig. 7b, whereas some of the simulated extinction spectra are reported in Supplementary Fig. 7c). The consistent agreement between the experimental extinction spectrum and that worked out by FDTD provides assurance that the observed resonance profile is the result of the collective plasmonic behavior exhibited by a $2 \mathrm{D}$ array made of spherical AuNPs, and that the quite large experimental spectral broadening also arises from the partial reduction of the degree of the spatial order due to the growth process.

Fluorescence-based immunoassay performance. The hybrid sandwich scheme shown in Fig. le was used in the 2D AuNP array functionalized by PIT for detecting PfLDH at ultra-low concentrations in one of the most complex matrices as it is the whole human blood. Two preliminary binding kinetic studies were performed in order to find out the incubation time necessary 
a

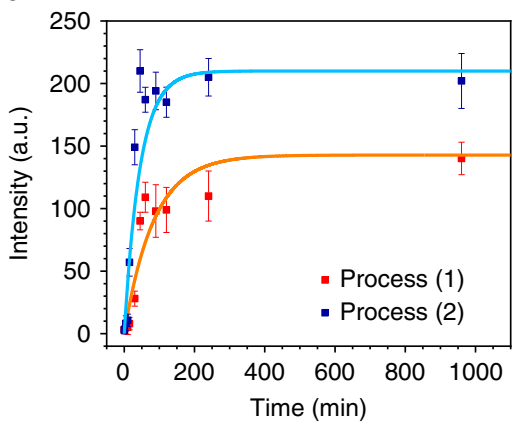

C

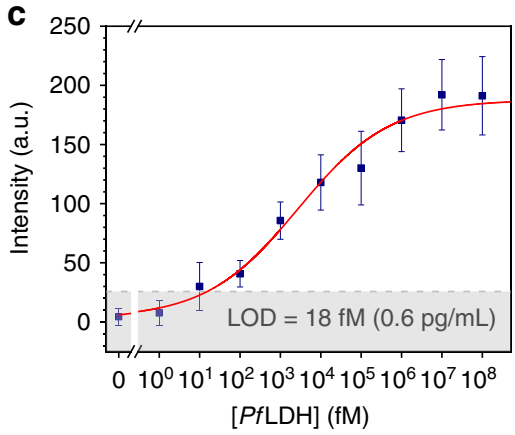

b

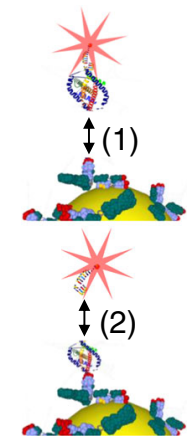

d

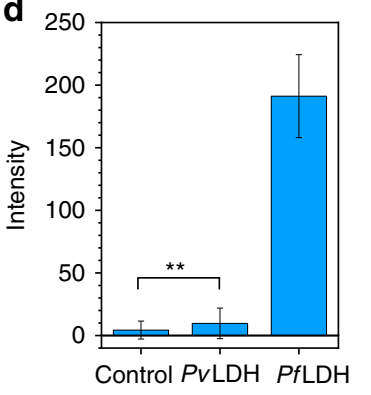

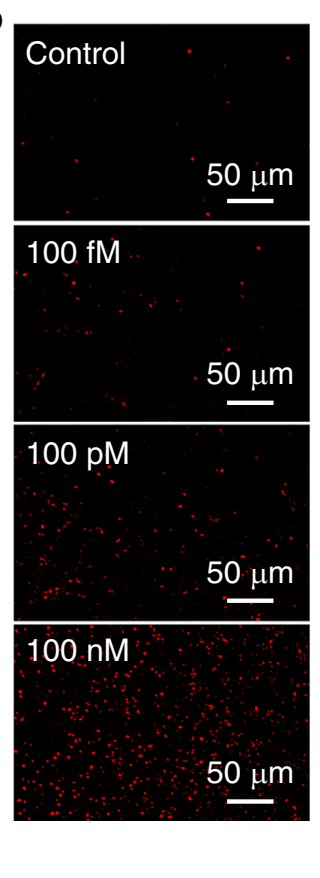

Fig. 4 Fluorescence-based immunoassay. a Kinetic curves related to (1) Ab-analyte and (2) analyte-aptamer binding dynamics. Both processes reach their equilibrium in a short time by incubation and tilting mixing ( $80 \mathrm{~min}$ and $45 \mathrm{~min}$, respectively). The time difference is ascribable to the significantly larger PfLDH mass. The data are well fitted by exponential curves (orange and blue continuous lines). b Fluorescence images acquired at different PfLDH concentrations in spiked human blood. c Calibration curve (fluorescence intensity vs PfLDH concentration in spiked human blood) of the immunoassay for PfLDH concentration in the range $1 \mathrm{fM}$ to $100 \mathrm{nM}$ ( $35 \mathrm{fg} / \mathrm{mL}$ to $3.5 \mu \mathrm{g} / \mathrm{mL}$ ). The data are best fitted by the four-parameter Hill equation (red solid line). The gray region represents the $3 \sigma$ noise level recorded in uncontaminated human blood (LOD $=18 \mathrm{fM}(0.6 \mathrm{pg} / \mathrm{mL}$ )). $\mathbf{d} \mathrm{Specificity}$ of the immunoassay against PVLDH (90\% residue identity with PfLDH) at a concentration of $100 \mathrm{nM}$ in spiked human blood. No cross-reaction detected with the main PfLD

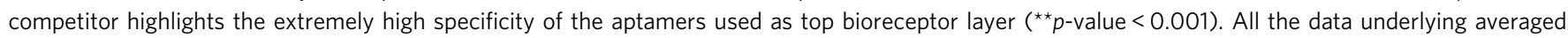
value are presented as mean value \pm SD and are representative of ten technical repeats. Source data are provided as a Source data file.

to reach the dynamic equilibrium for both (1) Ab-analyte and (2) analyte-aptamer binding. In order to detect the occurrence of the process (1), the analytes were made visible by complexing them with the fluorescently labeled aptamer by assuming that the small size of the aptamers did not significantly change the process kinetics, whereas as it concerns the process (2), fluorescent aptamers were incubated with $\mathrm{Ab}$-analyte complexes immobilized on the substrate. Both the processes (1) and (2) show very similar kinetics (Fig. 4a and Supplementary Fig. 8 for the corresponding fluorescence images) that are well fitted by exponential curves with a time constant of $80 \pm 30 \mathrm{~min}$ and $45 \pm 15 \mathrm{~min}$, respectively (orange and light blue continuous line in Fig. 4a). Since the fluorescence measurement could be carried out by commercial fluorescence readers, which are able to deliver multi-response within few minutes, the estimated detection time for the whole analysis is shorter than $3 \mathrm{~h}$. The slight difference in the fluorescence intensity at the dynamic equilibrium between the two kinetics can be ascribed to the pre-incubation among the free analytes and the fluorescent aptamers in the process (1), which leads to a less effective analyte binding since the aptamers might have targeted the PfLDH proteins on both sides, hindering their capture by $\mathrm{Abs}^{55}$.

Aiming at analyzing the whole blood, we had to dilute it to avoid clotting and turbidity of the solution to be incubated. The PfLDH was spiked into uninfected blood that was 1:100 diluted in $1 \mathrm{~mL}$ of $25 \mathrm{mM}$ Tris buffer achieving a good trade-off between high signal and treatable solution. Rather than exploiting additive concentrations, each substrate was used for a single concentration measurement so to inherently test the reliability and reproducibility of the detection procedure. Some of the resulting fluorescence images are shown in Fig. 4b (see Supplementary Fig. 9 for more images), from which one can observe that the number of the fluorescence spots is significantly higher than that the control down to a PfLDH concentration of $100 \mathrm{fM}$ $(3.5 \mathrm{pg} / \mathrm{mL})$. The intensity of a single spot turns out to be highly correlated to its area $A$ (see Supplementary Fig. 10) with an average area of $15 \mu^{2}$ corresponding to $\sim 36$ pixels (pixel area $=650 \times 650 \mathrm{~nm}^{2}$ ).

The FE factor is defined as $G=I_{\mathrm{PEF}} / I_{0}$, where $I_{\mathrm{PEF}}$ and $I_{0}$ are the fluorescence intensity from a single fluorophore with and without the plasmonic substrate, respectively. The latter measurement was realized by drop-casting $100 \mu \mathrm{L}$ of a solution containing $2.5 \mathrm{nM}$ of Cy5-aptamer complex on a bare microscope slide. The dried drop exhibited a quite circular shape with a diameter of $\sim 12 \mathrm{~mm}$, whereas the fluorescence imaging showed an irregular "coffee ring" whose thickness never exceeded $0.1 \mathrm{~mm}$. In order to assess the whole fluorescence intensity, the drop area was sampled in regions of interest, finding out that the intensity in the ring was 4 -fold higher than that of the inner region (see Supplementary Fig. 11). To determine the upper limit for the fluorescence intensity, we considered the emission area as given by a circle with $12 \mathrm{~mm}$ diameter and an outer annulus $0.1 \mathrm{~mm}$ thick obtaining a value $F_{0}=k_{\text {ins }} N_{0} I_{0}$, with $N_{0}$ the number of fluorophores in the drop $\left(1.5 \times 10^{11} \mathrm{Cy} 5\right.$-aptamer complexes $)$ and $k_{\text {ins }}$ an instrumental constant that includes the intensity of the incident radiation. In contrast with the previous case, the fluorescence with the plasmonic substrate consists of separated emitters (Fig. 4b); thus, if $N_{\mathrm{PEF}}$ is the number of spots, we have $F_{\mathrm{PEF}}=k_{\text {ins }} N_{\mathrm{PEF}} I_{\mathrm{PEF}}$. By measuring $F_{\mathrm{PEF}}$ with our $2 \mathrm{D}$ AuNP array, we obtained the lower limit $G=7 \times 10^{4}$ for the FE factor. 
Although the physics underlying the fluorescence enhancement by plasmonic nanostructures is still to be fully understood ${ }^{9,59}$, we can safely assess that such a high value of $G$ is also the result of the overlap of the plasmon resonance with both excitation and emission peaks ${ }^{13,14,17}$.

The fluorescence intensity $F$ as a function of the PfLDH concentration is reported in Fig. 4c. The experimental data are well fitted by the four-parameter Hill equation

$$
F([P f \mathrm{LDH}])=F_{1}+\frac{F_{2}-F_{1}}{1+\left(\frac{K}{[P f \mathrm{LDH}]}\right)^{n}}
$$

with $F_{1}=4 \pm 2$ arb. units, $F_{2}=190 \pm 10$ arb. units, $K=(2.7 \pm 1.4) \times$ $10^{3} \mathrm{fM}$, the Hill coefficient $n=0.38 \pm 0.08$ and $\chi^{2}=2$. The dynamic range extends over five decades (from $10 \mathrm{fM}$ to $1 \mathrm{nM}$ ), whereas a LOD of $18 \mathrm{fM}$ can be estimated by considering the signal threshold as three standard deviations of the control value (gray region in Fig. 4c).

The specificity of the biosensor is very challenging when the analyte has to be detected in a complex matrix such as human blood, but it is also particularly important for malaria diagnostics in areas where both $P$. falciparum and $P$. vivax are endemic as the clinical decision-making for drug treatment is different for infection with each species. In this respect, we studied the immunoassay response against the Plasmodium vivax lactate dehydrogenase $(P v \mathrm{LDH})$, which has a high functional and sequence similarity with PfLDH $\left(90 \%\right.$ residue identity $\left.{ }^{60}\right)$. The $P v \mathrm{LDH}$ was spiked into uninfected blood that was 1:100 diluted in buffer solution and the resulting fluorescence intensity is comparable to that measured from the control (Fig. 4d). Although the bottom anti-PLDH layer in our sandwich scheme captured any $P$ LDH malaria biomarkers, no significant crossreaction was detected in the case of $P v \mathrm{LDH}$ because of the extremely high specificity of the aptamers used as top bioreceptor layer. This is consistent with reports that the aptamer used in this assay is highly specific to PfLDH over $P v \mathrm{LDH}^{61}$.

\section{Discussion}

The gold standard for diagnosing malaria relies on the microscopic examination of blood films that not only necessitates trained personnel, but is unsuitable for a rapid diagnosis, which is required for a favorable prognosis of the disease ${ }^{62}$. Thus, big efforts are put in trying to develop rapid test based on a technology accessible even to the populations more exposed to the risk of infection. The most effective strategy largely adopted entails the detection of some of the enzymes expressed by the Plasmodium parasites that cause the disease. Lactate dehydrogenase is expressed in all Plasmodium species whilst an alternative biomarker, histidine rich protein II (HRP2), is only expressed in $P$. falciparum. Typically, for $P$. falciparum detection, LDH is preferred to HRP since the former better correlates with the parasite density ${ }^{41}$, however often species-specific detection requires tests against both $\mathrm{LDH}$ and HRP. This work describes an immunosensor capable to specifically detect $P f \mathrm{LDH}$ in whole blood at femtomolar level (LOD $<1 \mathrm{pg} / \mathrm{mL})$, which is a limit of detection several orders of magnitude lower than the rapid diagnostic tests ${ }^{63}$ or the commercial ELISA kits ${ }^{41,64}$. Such a LOD is reached thanks to a unique optimization of two ingredients of a biosensor: the transduction mechanism and the surface biofunctionalization, coupled with the recognition of the biomarker by both antibodies and aptamers. Furthermore, the functionalization procedure of PIT can be carried out by a simple UV lamp in a few minutes, thus we anticipate that such an approach can be carried out in low resource settings which would be critical for many malaria diagnostic applications. Therefore, we foresee that our approach may provide a solution to the cold transport challenges common to the presently used immunochromatographic lateral flow assays ${ }^{45}-47$.

Whilst the PfLDH and $P v \mathrm{LDH}$ investigated for the data herein were expressed in E. coli, previously solved crystal structures of these proteins in complex with aptamers show that these proteins do reflect the native conformation of these proteins ${ }^{55,65}$. It has been highlighted that sensitivity is a critical issue for malaria rapid diagnostic tests. The limit of detection for $P L D H-b a s e d$ immunochromatographic rapid diagnostic tests are typically in the range of $25 \mathrm{ng} / \mathrm{mL}^{63}$. The approach we have developed herein is over 1000-fold more sensitive. This opens up the possibility of point-of-care detection of $P \mathrm{LDH}$ in saliva which could transform malaria diagnosis as then one could diagnose non-invasively without the need for drawing blood from a patient. Such an approach would also reduce the risk of transmitting blood-borne pathogens. PLDH has been demonstrated to be present in saliva ${ }^{66}$, but there is no technology yet developed with sufficient sensitivity. Attempts to diagnose in saliva using conventional approaches lead to a high number of false negatives ${ }^{46}$. The ultrasensitive approach we describe herein may provide a costeffective solution providing the first non-invasive approach for a point-of-care diagnosis of malaria. A clinical study will be required to address this possibility.

Regarding transduction, we exploited the enhancement of the fluorescence intensity occurring by means of plasmonic nanostructures. Such a process is critically dependent on a number of conditions, the most noticeable being (i) the geometry of the nanostructure, (ii) the amount of overlap between the plasmonic resonance with both the fluorophore absorption and emission and (iii) the distance between the fluorophore and the surface. The constraint of realizing a device for practical applications suggested us to adopt the $\mathrm{BCMN}$, which allows one to rapidly fabricate ordered arrays of gold nanoparticles over large surfaces ${ }^{33}$, readily usable as a substrate for multiwell plates. In order to choose optimal values for the diameter $D$ and the inter-particle distance $d$, we fixed their ratio as $D / d=2.5-$ so to have significant interaction among the gold nanoparticle-and examined how the electric field intensity distribution varies as a function of nanoparticle size. Numerical simulations showed that the maximum amplification of the incident field is achieved at $D=50 \mathrm{~nm}$ and $d=20 \mathrm{~nm}$, a configuration hardly realizable by conventional techniques.

Since the nanoparticles are realized by a growing process, their interparticle distribution has a standard deviation of $14 \mathrm{~nm}$, whereas their surface shows some degree of roughness. Rather than being a drawback, such features lead to a relatively wide plasmon resonance peak that realizes the so-called dual enhancement mechanism ${ }^{19}$, in which the plasmon resonance overlaps with both the excitation and emission spectra of the fluorophore (point ii). The fluorescence enhancement only occurs when the fluorophore is placed at approximately $10 \mathrm{~nm}$ from the surface (point iii) ${ }^{14,16}$. To achieve precise positioning of the fluorophore, we exploited an effective surface biofunctionalization procedure (PIT), which binds the Abs so that their Fab region is at $\sim 5 \mathrm{~nm}$ from the surface 39,67 , with a sandwich scheme that included a tagged aptamer on the top. Since the size of both the $P f L D H$ and the aptamer is only a few nanometers, the fluorophore automatically positions itself at the optimal distance from the surface, and this was demonstrated by the measuredexperimental enhancement factor of $7 \times 10^{4}$ of the system.

As previously shown, beside oriented antibodies, the surface functionalization adopted in this work leads also to their close packed distribution both on AuNPs ${ }^{52,53}$ and flat surfaces ${ }^{68}$. In this way, not only the interacting area of the biosensor is fully exploited thereby obtaining high sensitivity and low LOD, but also the specificity is inherently warranted by the presence of the antibodies (and even more increased in our scheme by the 
presence of the aptamers). As a result of the occurrence of optimal conditions for detecting PfLDH, we were able to measure a LOD of $18 \mathrm{fM}(0.6 \mathrm{pg} / \mathrm{mL})$, which is comparable to that recently measured by means of a much more complex Luminex technology ${ }^{69}$.

By building on our results, two straightforward perspectives can be explored and even combined to make the clinical potential of our approach of significant impact: multiplexed and ultrasensitive (at attomolar level) detection. The former can be accomplished by functionalizing each section of a large substrate (e.g., multiwell plate) with different antibodies so that several analytes can be easily distinguished by their position on the substrate. On the other hand, detection at the attomolar level can be achieved by detecting the analyte in serum or plasma since these matrices would not require the 1:100 dilution carried out in this work to reduce the turbidity of the whole blood.

\section{Methods \\ Materials and chemicals. Diblock copolymers (P18226-S2VP) were purchased} from Polymer Source Inc. (Dorval, Canada) and were made by polystyrene $(x)$-b-2poly-vinylpyridine $(y)(\mathrm{PS}(x)-\mathrm{b}-\mathrm{P} 2 \mathrm{VP}(y))$, in which $x=30,000 \mathrm{~g} / \mathrm{mol}$ and $y=$ $8500 \mathrm{~g} / \mathrm{mol}$ are the molecular weight of polystyrene (PS) and poly(2-vinylpyridine) (P2VP), respectively. Toluene $(99.8 \%)$, gold(III) chloride trihydrate

$\left(\mathrm{HAuCl}_{4} \cdot 3 \mathrm{H}_{2} \mathrm{O}\right)$, silver nitrate $\left(\mathrm{AgNO}_{3}\right)$ and ascorbic acid were purchased from Sigma-Aldrich; acetone ( $\geq 99.0 \%)$, 2-propanol $(\geq 99.5 \%)$ and ethanol $(\geq 99.5 \%)$ were purchased from Merck Millipore; hexadecyltrimethylammonium bromide (CTAB) $(\geq 99.0 \%)$ was purchased from Fluka; bovine serum albumin (BSA) (fraction V IgG free, fatty acid poor) was obtained from Gibco. Ultrapure deionized water used for all aqueous solutions was dispensed by Milli- $\mathrm{Q}^{\oplus}$ system $(18.2 \mathrm{M} \Omega \mathrm{cm}$ resistivity). $10 \mathrm{mM}$ phosphate-buffered saline (PBS) $\left(\mathrm{NaCl} 10 \mathrm{mM}, \mathrm{NaH}_{2} \mathrm{PO}_{4} 10 \mathrm{mM}\right.$, $\left.\mathrm{Na}_{2} \mathrm{HPO}_{4} 10 \mathrm{mM}, \mathrm{MgCl}_{2} 1 \mathrm{mM}, \mathrm{pH} 7.1\right)$ and $25 \mathrm{mM}$ Tris-HCl buffer $(\mathrm{NaCl}$ $100 \mathrm{mM}$, imidazole $20 \mathrm{mM}$, Tris $25 \mathrm{mM}$, HCl $25 \mathrm{mM}$, pH 7.5) were prepared by dissolving the reagents (purchased from Sigma-Aldrich) in ultrapure water. Pan malaria antibody (monoclonal anti- $P$ LDH antibody clone $19 \mathrm{~g} 7$ ) was produced by Vista Laboratory Services (Langley, USA). The recombinant Plasmodium falciparum lactate dehydrogenase (PfLDH) and Plasmodium vivax lactate dehydrogenase $(P v \mathrm{LDH})$ were obtained from bacterial expression as described previously ${ }^{61}$. The malaria 2008 s aptamer labeled with cyanine 5 tag $\left(5^{\prime}\right.$-Cy5-CTG GGC GGT AGA ACC ATA GTG ACC CAG CCG TCT AC-3') was synthesized by Friz Biochem $\mathrm{GmbH}$ (Neuried, Germany). Millex syringe filters (pore size $0.20 \mu \mathrm{m}$ ) with hydrophilic polytetrafluoroethylene membrane were purchased from Merck Millipore; Superslip ${ }^{\mathrm{TM}}$ coverslips (borosilicate glass, thickness $0.13-0.17 \mathrm{~mm}$ ) were purchased from Thermo Fisher Scientific and cut by diamond tipped glass cutter.

Fabrication of 2D AuNP array. $29.2 \mathrm{mg}$ of diblock copolymer P18226-S2VP was added into $15 \mathrm{~mL}$ of toluene under vigorous stirring and controlled conditions (argon inert gas, $\mathrm{O}_{2}<1 \mathrm{ppm}, \mathrm{H}_{2} \mathrm{O}<0.1 \mathrm{ppm}$ ) for $72 \mathrm{~h}$ achieving homogeneously dispersed reverse micelle. Then, $15.7 \mathrm{mg}$ of $\mathrm{HAuCl}_{4} \cdot 3 \mathrm{H}_{2} \mathrm{O}$ was loaded in the solution by holding the vigorous stirring for $72 \mathrm{~h}$ allowing the gold precursor to be housed into the hydrophilic core of the micelles. When the gold powder was completely dispersed, the yellowish solution was filtered to remove micelle aggregates and impurities. The solution can be stored by holding the vigorous stirring up to approximately six months. Before depositing the PS-AuNPs on the glass coverslips $\left(10 \times 8 \mathrm{~mm}^{2}\right)$, the substrates were cleaned by sonication for $5 \mathrm{~min}$ in acetone, 2-propanol and pure ethanol sequentially, and dipped in toluene in order to make non-polar the surface so that the hydrophobic shells could stick on that. Then, the glass coverslips were dipped into the PS-AuNPs solution by dipcoater to carefully tune the dipping speed. A dipping speed of $0.6 \mathrm{~mm} / \mathrm{s}$ provided an optimal coverage of the glass surface in terms of both PS-AuNPs close-packing and long-range regularity (see Supplementary Fig. 6). The copolymers were etched by oxygen plasma treatment $(0.8 \mathrm{mbar}$ pressure, $200 \mathrm{~W}$ power, $30 \mathrm{~min})$ leaving the AuNPs immobilized on the glass surface at prefixed positions. Afterward, the substrates were incubated with $2 \mathrm{~mL}$ gold growth solution (CTAB $190 \mathrm{mM}$, $\mathrm{HAuCl}_{4} \cdot 3 \mathrm{H}_{2} \mathrm{O} 42 \mathrm{mM}, \mathrm{AgNO}_{3} 8 \mathrm{mM}$, ascorbic acid $100 \mathrm{mM}$ ) for $2 \mathrm{~h}$ in dark condition. Then, the substrates were copiously rinsing by ultrapure water and stored in dark condition until use.

Substrate biofunctionalization and blocking. The functionalization of AuNPs with anti-PLDH was achieved by PIT. $1 \mathrm{~mL}$ aqueous solution of anti-PLDH $(50 \mu \mathrm{g} / \mathrm{mL})$ was irradiated by an UV-lamp (Trylight ${ }^{\oplus}$, Promete S.r.l.) for $30 \mathrm{~s}$ and then flowed onto the substrate. The UV source consisted of two U-shaped lowpressure mercury lamps ( $6 \mathrm{~W}$ at $254 \mathrm{~nm}$ ) in which a standard quartz cuvette could be easily housed (Fig. 1b). By considering the wrapping geometry of the lamps and the proximity of the cuvette, the irradiation intensity used for the thiol group production was approximately $0.3 \mathrm{~W} / \mathrm{cm}^{2}$. Such an intensity is low enough to avoid any significant photolysis of the disulfide bridge that poorly absorbs at $254 \mathrm{~nm}^{70}$. Then, the substrates were rinsed by ultrapure water to remove the unbound Abs. As it concerns the blocking of the free active surface, $1 \mathrm{~mL}$ of BSA aqueous solution $(50 \mu \mathrm{g} / \mathrm{mL})$ was used to prevent nonspecific adsorption. Afterward, the samples were copiously rinsed by ultrapure water and stored in PBS solution $(10 \mathrm{mM})$ at room temperature until use.

PfLDH capture by immobilized Abs. The desired amount of $P f \mathrm{LDH}$ was spiked into $1 \mathrm{~mL}$ of uninfected whole human blood (1:100 diluted in $25 \mathrm{mM}$ Tris buffer) The functionalized substrates were incubated with $1 \mathrm{~mL}$ of contaminated blood solution for $2 \mathrm{~h}$ at room temperature; a tilting laboratory shaker was used to improve the analyte diffusion. Then, the samples were copiously rinsed by Tris buffer $(25 \mathrm{mM})$ and ultrapure water to remove the unbound proteins.

\section{PfLDH targeting with fluorescently labeled aptamers. The samples were} transferred into $1 \mathrm{~mL}$ of PBS solution $(10 \mathrm{mM})$ containing $0.1 \mu \mathrm{M}$ of malaria $2008 \mathrm{~s}$ aptamers labeled with Cy5 tag. The solution was gently shaken for $2 \mathrm{~h}$ in dark condition by a tilting laboratory shaker so that the sandwich scheme reported in Fig. 1e is realized. Then, the samples were abundantly rinsed by PBS and ultrapure water to remove the unbound aptamers.

SEM image analysis. The substrate was observed by Zeiss LEO 1550VP field emission scanning electron microscope (FESEM) with a nominal resolution of $1 \mathrm{~nm}$ at $20 \mathrm{kV}$ acceleration voltage. The recorded SEM images (Supplementary Fig. 12a shows an example of raw image at high magnification) were processed by ImageJ software to retrieve information about the substrate morphology. To this aim, each image was binarized to isolate the objects from the background (Supplementary Fig. 12b) and segmented by "Watershed" tool implemented in Image to separate adjacent nanoparticles (Supplementary Fig. 12c). Then, object area $S$, perimeter $p$, shape descriptors (aspect ratio $A R$ and circularity $4 \pi S / p^{2}$ ) and centroid coordinates were measured by "Analyze Particles" tool implemented in ImageJ. Supplementary Fig. 12d shows an example of processed SEM image, in which the objects are decomposed in outlines (black line) and inner area (orange filling). Given the round shape of the objects (Supplementary Fig. 13), nanoparticle diameter was estimated as $D=2 \sqrt{S / \pi}$, whereas the centre-to-centre distance distribution was carried out by calculating the distance of each centroid from its nearest neighbors.

Processing and analysis of the fluorescence images. Fluorescence images were recorded by Zeiss Axio Observer Z1 inverted phase contrast fluorescence microscope equipped by Zeiss Colibri.2 LED light source (module $625 \mathrm{~nm}$ ), Zeiss PlanApochromat 10x/0.45 Ph1 M27 (FWD $=2.1 \mathrm{~mm})$ objective, cube 50 Cy5 filter (excitation $625-655 \mathrm{~nm} / \mathrm{emission} 665-715 \mathrm{~nm}$ ) and pco.edge 5.5 sCMOS photodetector (scaling $0.650 \mu \mathrm{m} \times 0.650 \mu \mathrm{m}$ per pixel, image size $2560 \times 2160$ pixels, scaled image size $1.66 \mathrm{~mm} \times 1.40 \mathrm{~mm}, 16$ bit dynamic range, $2 \mathrm{~s}$ exposure time was used for every image). The recorded fluorescence images were processed by ImageJ software. The "rolling ball" algorithm was used to remove the smooth continuous background from the images ${ }^{56}$ (see Supplementary Fig. S3). The background was locally measured for each pixel by averaging over a ball around the pixel. Such a value is then subtracted from the original image flattening the spatial variations of the background. The rolling ball radius was set 10 pixels, a length reasonably higher than the size of the largest objects that are not part of the background. A threshold level slightly higher than the flattened background was set to segment the image whose whole intensity was measured by summing the signal contribution of every spots. To ease the readability of the calibration curve (Fig. 4c), the intensities were scaled down by an arbitrary factor. Aiming at carrying out a robust and reliable analysis of the fluorescence signal, ten images were randomly recorded for each sample and the mean of their intensity was found out.

Simulations of the optical response. The optical response of both the 2D AuNP lattice and the actual substrate was simulated by "FDTD solutions" tool implemented in Lumerical software. A linearly polarized EM radiation traveling along the $z$ direction was used to investigate the system. Photodetectors (PDs) conveniently positioned in the workspace could measure the intensity of the EM field over time. A PD was dedicated to measure the extinction spectrum of the nanostructure. Symmetric/anti-symmetric boundary conditions (BCs) set along $x$ and $y$ direction extend the plasmonic response over an infinite 2D array while reduce the simulation time by a factor 8 without worsening the accuracy of the results. Bloch BCs were used only for polarization study in order to compensate the phase shift arising when an EM disturbance with a non-zero angle should be reinjected at the opposite workspace site. Perfect matched layer BCs set in $z$ assures perfect absorption of the EM waves backscattered through the plane containing the light source and incident upon the opposite side of the workspace. The workspace was discretized over a mesh with a spatial resolution of $0.5 \mathrm{~nm}$ and $1.0 \mathrm{~nm}$ for the 2D AuNP lattice and the actual substrate, respectively. Such a choice assured high accuracy while keeping the simulation time within few hours. The AuNPs of the lattice were modeled as homogeneous gold spheres ${ }^{71}$, while the bare substrate was represented as a thick dielectric layer of silicon dioxide $\left(\mathrm{SiO}_{2}\right)^{72}$. The extinction 
spectrum of the actual substrate was carried out by importing the SEM morphology in Lumerical workspace. To this aim, the raw SEM image (Supplementary Fig. 14a shows an example at high magnification) was thresholded and binarized to create a mask template, in which the object pixel value was set equal to 1 and the background to 0 (Supplementary Fig. 14b). The centroid coordinates $\left(i_{\mathrm{nc}}, j_{\mathrm{nc}}\right)$ of the $n^{\text {th }}$ object were retrieved by running "Analyze Particles" tool implemented in ImageJ software (Supplementary Fig. 14c). Following to the mask template, the centroid positions were used to shape spherical nanoparticles (diameter $D=50 \mathrm{~nm}$ ) as $z\left(i_{\mathrm{n}}, j_{\mathrm{n}}\right)= \pm \sqrt{(D / 2)^{2}-\left(i_{\mathrm{n}}-i_{\mathrm{nc}}\right)^{2}-\left(j_{\mathrm{n}}-j_{\mathrm{nc}}\right)^{2}}$ (Supplementary Fig. 14d).

Reporting summary. Further information on research design is available in the Nature Research Reporting Summary linked to this article.

\section{Data availability}

The data that support the findings of this study are available from the authors on reasonable request. Source data are provided with this paper.

\section{Code availability}

The codes used to analyse the data are available from the authors on reasonable request.

Received: 25 May 2020; Accepted: 8 October 2020;

Published online: 01 December 2020

\section{References}

1. Tran, N. H. T., Trinh, K. T. L., Lee, J.-H., Yoon, W. J. \& Ju, H. Reproducible enhancement of fluorescence by bimetal mediated surface plasmon coupled emission for highly sensitive quantitative diagnosis of double-stranded DNA. Small 14, 1801385 (2018).

2. Yousefi, H., Ali, M. M., Su, H.-M., Filipe, C. D. M. \& Didar, T. F. Sentinel wraps: real-time monitoring of food contamination by printing DNAzyme probes on food packaging. ACS Nano 12, 3287-3294 (2018).

3. Liu, P. et al. Fluorescence-enhanced bio-detection platforms obtained through controlled 'step-by-step' clustering of silver nanoparticles. Nanoscale 10, 848-855 (2018).

4. Markov, A. et al. Engineering of neuron growth and enhancing cell-chip communication via mixed SAMs. ACS Appl. Mater. Interfaces 10, 18507-18514 (2018).

5. Fehrenbacher, L. et al. NSABP B-47/NRG oncology phase III randomized trial comparing adjuvant chemotherapy with or without trastuzumab in high-risk invasive breast cancer negative for HER2 by FISH and with IHC $1+$ or 2. J. Clin. Oncol. 38, 444-453 (2020).

6. Michalet, X. et al. Quantum dots for live cells, in vivo imaging, and diagnostics. Science 307, 538-544 (2005).

7. Shima, T. \& Uemura, S. Molecular dynamics revealed by single-molecule FRET measurement. Make Life Visible 105-113, https://doi.org/10.1007/978981-13-7908-6_10 (2020).

8. Liang, Y., Huang, X., Yu, R., Zhou, Y. \& Xiong, Y. Fluorescence ELISA for sensitive detection of ochratoxin A based on glucose oxidase-mediated fluorescence quenching of CdTe QDs. Anal. Chim. Acta 936, 195-201 (2016).

9. Jeong, Y., Kook, Y.-M., Lee, K. \& Koh, W.-G. Metal enhanced fluorescence (MEF) for biosensors: general approaches and a review of recent developments. Biosens. Bioelectron. 111, 102-116 (2018).

10. Fothergill, S. M., Joyce, C. \& Xie, F. Metal enhanced fluorescence biosensing: from ultra-violet towards second near-infrared window. Nanoscale $\mathbf{1 0}$, 20914-20929 (2018).

11. Lakowicz, J. R. et al. Plasmon-controlled fluorescence: a new paradigm in fluorescence spectroscopy. Analyst 133, 1308 (2008).

12. Li, J. F., Li, C. Y. \& Aroca, R. F. Plasmon-enhanced fluorescence spectroscopy. Chem. Soc. Rev. 46, 3962-3979 (2017).

13. Chen, Y., Munechika, K. \& Ginger, D. S. Dependence of fluorescence intensity on the spectral overlap between fluorophores and plasmon resonant single silver nanoparticles. Nano Lett. 7, 690-696 (2007).

14. Li, M., Cushing, S. K. \& Wu, N. Plasmon-enhanced optical sensors: a review. Analyst 140, 386-406 (2015).

15. Bharadwaj, P. \& Novotny, L. Spectral dependence of single molecule fluorescence enhancement. Opt. Express 15, 14266 (2007).

16. Khurgin, J. B. \& Sun, G. Enhancement of optical properties of nanoscaled objects by metal nanoparticles. J. Opt. Soc. Am. B 26, B83 (2009).

17. Lakowicz, J. R. et al. Advances in surface-enhanced fluorescence. J. Fluoresc. 14, 425-441 (2004)

18. Geddes, C. D. \& Lakowicz, J. R. Metal-enhanced fluorescence. J. Fluoresc. 12, 121-129 (2002).
19. Knoblauch, R. \& Geddes, C. D. In Reviews in Plasmonics (ed. Geddes, C. D.) vol 2017, 253-283 (Springer International Publishing, 2019).

20. Della Ventura, B. et al. Biosensor for point-of-care analysis of immunoglobulins in urine by metal enhanced fluorescence from gold nanoparticles. ACS Appl. Mater. Interfaces 11, 3753-3762 (2019).

21. Usukura, E. et al. Highly confined, enhanced surface fluorescence imaging with two-dimensional silver nanoparticle sheets. Appl. Phys. Lett. 104, 121906 (2014).

22. $\mathrm{Du}, \mathrm{B}$. et al. Diameter-optimized high-order waveguide nanorods for fluorescence enhancement applied in ultrasensitive bioassays. Nanoscale 11, 14322-14329 (2019).

23. Xie, F., Centeno, A., Ryan, M. R., Riley, D. J. \& Alford, N. M. Au nanostructures by colloidal lithography: from quenching to extensive fluorescence enhancement. J. Mater. Chem. B 1, 536-543 (2013).

24. Pompa, P. P. et al. Metal-enhanced fluorescence of colloidal nanocrystals with nanoscale control. Nat. Nanotechnol. 1, 126-130 (2006).

25. Kinkhabwala, A. et al. Large single-molecule fluorescence enhancements produced by a bowtie nanoantenna. Nat. Photonics 3, 654-657 (2009).

26. Zhou, L. et al. Enhancement of immunoassay's fluorescence and detection sensitivity using three-dimensional plasmonic nano-antenna-dots array. Anal. Chem. 84, 4489-4495 (2012).

27. Flauraud, V. et al. In-plane plasmonic antenna arrays with surface nanogaps for giant fluorescence enhancement. Nano Lett. 17, 1703-1710 (2017).

28. Tabakman, S. M. et al. Plasmonic substrates for multiplexed protein microarrays with femtomolar sensitivity and broad dynamic range. Nat. Commun. 2, 466 (2011).

29. Zang, F. et al. Ultrasensitive Ebola virus antigen sensing via 3D nanoantenna arrays. Adv. Mater. 31, 1902331 (2019).

30. Puchkova, A. et al. DNA origami nanoantennas with over 5000 -fold fluorescence enhancement and single-molecule detection at $25 \mu \mathrm{M}$. Nano Lett. 15, 8354-8359 (2015)

31. Zhang, B., Kumar, R. B., Dai, H. \& Feldman, B. J. A plasmonic chip for biomarker discovery and diagnosis of type 1 diabetes. Nat. Med. 20, 948-953 (2014).

32. Luan, J. et al. Add-on plasmonic patch as a universal fluorescence enhancer Light Sci. Appl. 7, 29 (2018).

33. Glass, R., M ller, M. \& Spatz, J. P. Block copolymer micelle nanolithography. Nanotechnology 14, 1153-1160 (2003).

34. Ghosh, S. K. \& Pal, T. Interparticle coupling effect on the surface plasmon resonance of gold nanoparticles: from theory to applications. Chem. Rev. 107, 4797-4862 (2007).

35. Auguié, B. \& Barnes, W. L. Collective resonances in gold nanoparticle arrays. Phys. Rev. Lett. 101, 143902 (2008).

36. Toma, M. et al. Collective plasmon modes excited on a silver nanoparticle 2D crystalline sheet. Phys. Chem. Chem. Phys. 13, 7459 (2011).

37. Oliverio, M., Perotto, S., Messina, G. C., Lovato, L. \& De Angelis, F. Chemical functionalization of plasmonic surface biosensors: a tutorial review on issues, strategies, and costs. ACS Appl. Mater. Interfaces $\mathbf{9}$, 29394-29411 (2017).

38. Vashist, S. K. \& Luong, J. H. T. In Handbook of Immunoassay Technologies 19-46, https://doi.org/10.1016/B978-0-12-811762-0.00002-5 (Elsevier, 2018).

39. Della Ventura, B. et al. Biosensor surface functionalization by a simple photochemical immobilization of antibodies: experimental characterization by mass spectrometry and surface enhanced Raman spectroscopy. Analyst 144, 6871-6880 (2019).

40. Zhou, Y. et al. Fabrication of an antibody-aptamer sandwich assay for electrochemical evaluation of levels of $\beta$-amyloid oligomers. Sci. Rep. 6, 35186 (2016).

41. Waitumbi, J., Awinda, G., Rajasekariah, G.-H., Kifude, C. \& Martin, S. K. Unified parasite lactate dehydrogenase and histidine-rich protein ELISA for quantification of Plasmodium falciparum. Am. J. Trop. Med. Hyg. 80, 516-522 (2009).

42. Phillips, M. A. et al. Malaria. Nat. Rev. Dis. Prim. 3, 17050 (2017).

43. World Health Organization. World Malaria Report 2019. Geneva. (2019).

44. Snow, R. W. Global malaria eradication and the importance of Plasmodium falciparum epidemiology in Africa. BMC Med. 13, 23 (2015).

45. Bell, D., Jorgensen, P., Tsuyuoka, R., Chanthap, L. \& Rebueno, A. Malaria rapid diagnostic tests in tropical climates: the need for a cool chain. Am. J. Trop. Med. Hyg. 74, 750-754 (2006).

46. Nambati, E. A. et al. Unclear association between levels of Plasmodium falciparum lactate dehydrogenase (PfLDH) in saliva of malaria patients and blood parasitaemia: diagnostic implications? Malar. J. 17, 9 (2018).

47. Lee, W. S. et al. Simple, rapid, and accurate malaria diagnostic platform using microfluidic-based immunoassay of Plasmodium falciparum lactate dehydrogenase. Nano Converg. 7, 13 (2020).

48. Lee, W., Lee, S. Y., Briber, R. M. \& Rabin, O. Self-assembled SERS substrates with tunable surface plasmon resonances. Adv. Funct. Mater. 21, 3424-3429 (2011). 
49. Della Ventura, B., Schiavo, L., Altucci, C., Esposito, R. \& Velotta, R. Light assisted antibody immobilization for bio-sensing. Biomed. Opt. Express 2, 3223 (2011).

50. Funari, R. et al. Single molecule characterization of UV-activated antibodies on gold by atomic force microscopy. Langmuir 32, 8084-8091 (2016).

51. Neves-Petersen, M. T. et al. High probability of disrupting a disulphide bridge mediated by an endogenous excited tryptophan residue. Protein Sci. 11 588-600 (2009).

52. Della Ventura, B. et al. Effective antibodies immobilization and functionalized nanoparticles in a quartz-crystal microbalance-based immunosensor for the detection of parathion. PLOS ONE 12, e0171754 (2017).

53. Iarossi, M. et al. Colorimetric immunosensor by aggregation of photochemically functionalized gold nanoparticles. ACS Omega 3, 3805-3812 (2018).

54. Low, Y. K. et al. Development of an ultrasensitive impedimetric immunosensor platform for detection of plasmodium lactate dehydrogenase. Sensors 19, 2446 (2019).

55. Cheung, Y.-W. et al. Structural basis for discriminatory recognition of Plasmodium lactate dehydrogenase by a DNA aptamer. Proc. Natl Acad. Sci. USA 110, 15967-15972 (2013).

56. Sternberg, S. R. Biomedical image processing. Computer (Long. Beach Calif.) 16, 22-34 (1983).

57. Ross, M. B., Mirkin, C. A. \& Schatz, G. C. Optical properties of one-, two-, and three-dimensional arrays of plasmonic nanostructures. J. Phys. Chem. C. 120, 816-830 (2016).

58. Mujumdar, R. B., Ernst, L. A., Mujumdar, S. R., Lewis, C. J. \& Waggoner, A. S. Cyanine dye labeling reagents: sulfoindocyanine succinimidyl esters. Bioconjug. Chem. 4, 105-111 (1993).

59. Lucas, E., Knoblauch, R., Combs-Bosse, M., Broedel, S. E. \& Geddes, C. D. Low-concentration trypsin detection from a metal-enhanced fluorescence (MEF) platform: Towards the development of ultra-sensitive and rapid detection of proteolytic enzymes. Spectrochim. Acta Part A Mol. Biomol. Spectrosc. 228, 117739 (2020).

60. Turgut-Balik, D. et al. Cloning, sequence and expression of the lactate dehydrogenase gene from the human malaria parasite, Plasmodium vivax. Biotechnol. Lett. 26, 1051-1055 (2004).

61. Cheung, Y.-W. et al. Aptamer-mediated Plasmodium-specific diagnosis of malaria. Biochimie 145, 131-136 (2018).

62. Mathison, B. A. \& Pritt, B. S. Update on malaria diagnostics and test utilization. J. Clin. Microbiol. 55, 2009-2017 (2017).

63. Jimenez, A. et al. Analytical sensitivity of current best-in-class malaria rapid diagnostic tests. Malar. J. 16, 128 (2017)

64. Kifude, C. M. et al. Enzyme-linked immunosorbent assay for detection of Plasmodium falciparum histidine-rich protein 2 in blood, plasma, and serum. Clin. Vaccin. Immunol. 15, 1012-1018 (2008).

65. Cheung, Y.-W. et al. Evolution of abiotic cubane chemistries in a nucleic acid aptamer allows selective recognition of a malaria biomarker. Proc. Natl Acad. Sci. USA 117, 16790-16798 (2020).

66. Gbotosho, G. O. et al. Rapid detection of lactate dehydrogenase and genotyping of Plasmodium falciparum in saliva of children with acute uncomplicated malaria. Am. J. Trop. Med. Hyg. 83, 496-501 (2010).

67. Minopoli, A. et al. LSPR-based colorimetric immunosensor for rapid and sensitive $17 \beta$-estradiol detection in tap water. Sens. Actuators B Chem. 308, 127699 (2020).

68. Cimafonte, M. et al. Screen printed based impedimetric immunosensor for rapid detection of Escherichia coli in drinking water. Sensors 20, 274 (2020).

69. Martiáñez-Vendrell, X. et al. Quantification of malaria antigens PfHRP2 and pLDH by quantitative suspension array technology in whole blood, dried blood spot and plasma. Malar. J. 19, 12 (2020).
70. Neves-Petersen, M. T. et al. Flash Photolysis of Cutinase: Identification and Decay Kinetics of Transient Intermediates Formed upon UV Excitation of Aromatic Residues. Biophys. J. 97, 211-226 (2009).

71. Haynes, W. M. CRC Handbook of Chemistry and Physics. CRC Handbook of Chemistry and Physics, https://doi.org/10.1201/9781315380476 (CRC Press, 2016).

72. Palik, E. D. Handbook of Optical Constants of Solids. Handbook of Optical Constants of Solids https://doi.org/10.1016/C2009-0-20920-2 (Elsevier, 1985).

\section{Acknowledgements}

We would like to thank Ruoyan Wei for her help on substrate fabrication through block copolymer micelle nanolithography and Gabriela Figueroa Miranda for her advices on aptamer handling.

\section{Author contributions}

A.M., B.DV., R.V., and D.M. conceived the project. A.M. carried out the experiments and collected the data under D.M. and A.O. supervision and administration. A.M., B.L., and F.G. worked out the numerical simulations. A.M., B.DV., R.V., and F.G. performed the data interpretation. J.A.T. and D.M. provided support on the use of aptamers. A.M., R.V., and J.A.T. wrote the paper. All authors were involved in the revisions.

\section{Funding}

Open Access funding enabled and organized by Projekt DEAL.

\section{Competing interests}

The authors declare no competing interests.

\section{Additional information}

Supplementary information is available for this paper at https://doi.org/10.1038/s41467 020-19755-0.

Correspondence and requests for materials should be addressed to D.M. or R.V.

Peer review information Nature Communications thanks Andrew Kirk, Alfredo Mayor and the other anonymous reviewers for their contribution to the peer review of this work. Peer reviewer reports are available.

Reprints and permission information is available at http://www.nature.com/reprints

Publisher's note Springer Nature remains neutral with regard to jurisdictional claims in published maps and institutional affiliations.

\begin{abstract}
(c) Open Access This article is licensed under a Creative Commons C. Attribution 4.0 International License, which permits use, sharing, adaptation, distribution and reproduction in any medium or format, as long as you give appropriate credit to the original author(s) and the source, provide a link to the Creative Commons license, and indicate if changes were made. The images or other third party material in this article are included in the article's Creative Commons license, unless indicated otherwise in a credit line to the material. If material is not included in the article's Creative Commons license and your intended use is not permitted by statutory regulation or exceeds the permitted use, you will need to obtain permission directly from the copyright holder. To view a copy of this license, visit http://creativecommons.org/ licenses/by/4.0/.
\end{abstract}

(C) The Author(s) 2020 